\title{
EDITORIAL
}

\section{Molecular Psychiatry: 20 Years}

\author{
Molecular Psychiatry (2015) 20, 545-547; doi:10.1038/mp.2015.25; \\ published online 17 March 2015
}

Twenty years ago we had the idea to start a new journal that would catalyze the emerging field of molecular psychiatry. ${ }^{1}$ At the time, some questioned the journal title and wondered whether it was an oxymoron. Our field has grown immensely in the past 20 years, and today, of course, the use of 'molecular' alongside 'psychiatry' is fully accepted. Not only that, Molecular Psychiatry's impact factor has been the highest among psychiatry journals each year for the last five years. ${ }^{2}$ Yet the translation of our work to improved clinical outcomes for patients with psychiatric disorders is still in the realm of promise. The major difference, as we see it, is that what was a faraway destination now seems to be tantalizing close, temptingly around the corner, almost within reach.

There are pessimistic and optimistic ways to look at our field now. Pessimistically, one could say that the gulf between knowledge and its application to improve people's lives has never been greater. In the past 20 years, new knowledge gained in psychiatry and psychology has not resulted in conceptually novel treatments that have reached health-care delivery streams. Both our pharmacological and psychological treatments are conceptually old, bordering on the archaic. Optimistically, it could be stated that the amount of knowledge generated in the past 20 years-a substantial portion of which was published in this journal-has been truly amazing and that we are well poised to make major translational advances. If that is indeed the case, it would be appropriate to consider what the next 20 years might bring.

Although some have strongly advocated that research should focus solely on identifying new treatment strategies, we believe that an optimal and balanced approach to revenue expenditure in psychiatry should combine support for efforts aimed at improving existing treatments with support for efforts to identify new leads and truly novel treatment approaches.

Pharmacogenomics, now being rebranded as 'precision medicine', is a major area for progress, ${ }^{3}$ and it has been recognized as a priority. In his State of the Union Address on 20 January 2015, US President Barack Obama briefly announced the Precision Medicine Initiative-unveiled on 30 January 2015 as 'a bold new research effort to revolutionize how we improve health and treat disease'. Launched with a \$215 million investment in the president's 2016 budget, the Precision Medicine Initiative will pioneer a model of patient-powered research that promises to accelerate biomedical discoveries and provide clinicians with new tools, knowledge, and therapies to help them determine which treatments will work best for which patients. Most medical treatments have been designed for the 'average patient'. One-size-fits-all-approach treatments can be very successful for some patients but not for others. This is changing with the emergence of precision medicine, an innovative approach to disease prevention and treatment that takes into account individual differences in people's genes, environments, and lifestyles. Precision medicine gives clinicians tools to better understand the complex mechanisms underlying a patient's health, disease, or condition, and to better predict which treatments will be most effective. The centerpiece of the Precision Medicine Initiative will be $\$ 130$ million for the development of 'a voluntary national research cohort of a million or more volunteers to propel our understanding of health and disease and set the foundation for a new way of doing research through engaged participants and open, responsible data sharing. ${ }^{4}$ We wholeheartedly applaud this outstanding and timely initiative.

While we recognize that medicine will, for the foreseeable future, not be $100 \%$ precise, there is certainly room for greatly enhancing clinical practice through the prediction of outcomes based on the tools of genomics and other -omics, such as transcriptomics, proteomics, lipidomics, and metabolomics. At present, in psychiatry each treatment is an $n$ of 1 clinical study, in which efficacy cannot be predicted. As is the case in all of medicine, our patients tend to respond to some but not all treatments and typically have an adverse drug reaction (ADR) profile that varies from drug to drug, some being well tolerated and others not. The outcomes of treatment with the same drug, administered for what appear to be similar clinical presentations that are grouped together under the same DSM-5 (Diagnostic and Statistical Manual of Mental Disorders, Fifth Edition) diagnosis, may vary widely, from full remission without significant ADRs to lack of response or partial response with or without ADRs. Clinically, the most challenging outcomes consist of a partial clinical response with moderate side effects. The decision as to whether to stop or modify treatment, and to what degree, can result in either clinical improvement or deterioration and relapse. Such decisions are currently made in an uninformed manner, with no tools or markers of outcomes that can be used as a guide.

Psychotropic drugs are among the best-selling drug classes. This includes antipsychotics and antidepressants, the latter being the second most prescribed drug class in the United States. The most prescribed class is analgesics, which includes over-thecounter products such as aspirin and acetaminophen. Therefore, of prescribed drug classes, antidepressants are the top sellers: $11 \%$ of the US population over 12 years of age is on antidepressants. It is just not acceptable in the light of existing technologies that a major effort is not being made to identify and validate markers that would enable clinicians to target specific treatments on an individual basis. Without using any new products, psychiatric treatment would be greatly enhanced if we could use existing medications in conjunction with tools to predict positive response as well as ADRs.

A major roadblock for pharmacogenomics is cost. After all, pharmacogenomics is a branch of pharmacology, which is a very precise science. In clinical pharmacological studies, including those in the area of pharmacogenomics, drugs must be given in consistent doses over specified periods of time, and outcomes must be documented prospectively, in standardized fashion. It would be unthinkable to do a retrospective pharmacokinetics study without precise data. Unfortunately, in the field of pharmacogenomics that has been the case. Very few studies have been done in a prospective manner, using a double-blind design and placebo. ${ }^{5}$ Because such studies can include only relatively small numbers of subjects, new approaches to data analysis are needed, including methods that have been recently proposed. ${ }^{6}$ Researchers collect samples of convenience from patients who have undergone standard-of-care treatment (in most cases, taking a variety of drugs and doses), then collect, in a nonstructured manner, retrospective treatment outcome data, which are notoriously unreliable. Those very messy and unreliable results are then correlated with complex genomic data, yielding, obviously, outcomes that are variable, not often replicable, and unlikely to lead to effective translation into practice. In order to 
advance, this field needs abundant and consistent funding to cover the costs of recruiting and treating, in a standardized manner, large numbers of people who can then be subjected to testing for genomic and other -omics predictors of treatment response. Such studies are large, costly, and not particularly creative, innovative, or revolutionary, and they will not fare well in typical review panels for government medical research grants, unless there are specific calls for applications in this area. However, without such new research, treatment with existing tools will remain a costly and ineffective guessing game that is of limited benefit to individuals with psychiatric disorders. We hope that in 20 years, as limitations to research in this area are overcome, and possibly as the Precision Medicine Initiative comes to fruition, we will have arrived at a time when $a$ priori testing will lead to treatment that is targeted for efficacy and tolerance.

Another area that needs to be further developed in pharmacological research is the long-term outcomes of treatment. While much research has been directed at potentially severe side effects of antidepressants, ${ }^{7,8}$ the assumption has been that once treatment ends, drug effects are no longer present. That is not necessarily the case. We developed a novel animal paradigm consisting of short-term exposure to stress and antidepressants followed by a long-term high-fat diet. Using this model, we found that recurrent restraint stress-related weight loss was recovered 2 weeks after the end of stress in young growing rats fed a high-fat diet. When exposed to a high fat-diet after stress, even months (17-22 weeks) after antidepressant treatment had ended, animals showed significant increases in caloric intake, body weight, and size as compared with control animals treated with saline and fed the same high-fat diet. ${ }^{9}$ Our own data are consistent with the previously described phenomenon of time-dependent sensitization ${ }^{10}$ and support the notion that enduring effects of short-term antidepressant treatment become manifest on a long-term basis after antidepressant discontinuation, during conditions of high stress followed by high-fat intake. We suggested that antidepressant exposure might be a covert, insidious, and enduring risk factor for obesity and for the obesity epidemic, even after discontinuation of antidepressant treatment. Much research needs to be done in this area. It is necessary to investigate the long-term effects (after discontinuation) of other types of widely used psychotropic drugs, such as atypical antipsychotics, stimulants and other drugs used for attention-deficit hyperactivity disorder, anxiolytics, and other drug classes. Thankfully, we now have the tools to dissect mechanisms for such protracted effects. Those tools include the ability to look at DNA methylation across the entire genome. It is clinically imperative to determine whether psychotropic medications cause DNA methylation resulting in long-term effects, even after drug discontinuation, which can be unmasked by environmental factors.

Diagnosis will greatly benefit from further research. In order for knowledge to be advanced in the field of psychiatry, a classification system must exist to group patients based on defining characteristics. Existing classifications, such as the International Classification of Diseases (ICD)-10 and the DSM-5, (ref. 11) are still conceptually based on decades-old recommendations from the World Health Organization (WHO) for an atheoretical classification based entirely on descriptively grouping clusters of symptoms. ${ }^{12}$ Whereas the WHO recommendation was specific for epidemiological studies, both ICD-10 and DSM-IV and 5 have been extensively used for clinical diagnostic purposes, for health insurance and reimbursement, and to guide research. As modern medicine has evolved since 1959, it has become evident that the same causal mechanisms can result in diverse clinical presentations, and different groups of contributing factors can result in similar presentations. It is highly likely that clusters of symptoms-for example, the ones we now know as major depression, bipolar disorder, and schizophrenia-will ultimately prove to be final common manifestations of diverse groupings of causal factors. Conversely, it is expected that the same variants of genes relevant to brain function will, in different individuals, result in different types of clinical presentations.

To break away from this classification conundrum, the National Institute of Mental Health has developed a conceptually novel approach, the Research Domain Criteria (RDoC) project, ${ }^{13-19}$ aimed at providing a logical transition from genes to molecules, cells, circuits, physiology, behaviors, self-reports, and potential clinical paradigms. That approach is based on domains of behavior for which a biological underpinning can be sought. In 20 years' time, this approach should have been extensively tested and at that point we would then have a science-based approach to psychiatric diagnoses.

Based on trends provided by current data, it appears that the genetic basis of psychiatric disorders consists of multiple common variants of very small effect. Particularly for disorders such as autism, there can also be de novo mutations. It still needs to be determined what, if any, role rare or de novo mutations will have in terms of a major contribution to psychiatric disorders such as schizophrenia, bipolar disorder, or major depression.

Other areas of long-standing interest that are becoming increasingly relevant include the role of peripheral mediators in brain function and dysfunction as seen in psychiatric disorders. These include inflammatory mediators and adipokines, ${ }^{20-23}$ as well as the microbiome. ${ }^{24,25}$

One exciting feature of our twentieth anniversary, and hopefully of our next 20 years, is our partnership with the American Journal of Psychiatry in hosting a joint symposium at the Annual Meeting of the American Psychiatric Association in Toronto, Ontario, Canada. Entitled 'Breaking New Research from the American Journal of Psychiatry on Adolescent Drinking and from Molecular Psychiatry on both Schizophrenia and Suicide', this symposium, to be held 15 May 2015, will highlight two papers from the American Journal of Psychiatry and two papers from Molecular Psychiatry. Chosen from our most groundbreaking work, the two Molecular Psychiatry articles are currently under embargo before their May 2015 release. Broadly speaking, the papers will (i) reveal a novel treatment strategy for schizophrenia and (ii) provide new data on suicidality and mental disorders, obtained from a national, prospective study. We are excited that such innovative work has found a home in our journal and that those original research articles will be highlighted at the largest psychiatric meeting in the world.

The danger with predicting the future is that it is mostly a futile exercise. As children and adolescents, we loved science fiction. Of all the astounding features predicted for life in the twenty-first century, the vast majority have not come to pass. There are no space stations, flying cars, or domestic robots. Not all predictions failed to materialize, however: one item that appeared in the sci-fi of our youth and became a reality was the mobile phone. But the Internet, which is now an essential tool, cannot be found in any science fiction work of the mid-twentieth century. Therefore, rather than further indulging in what the next 20 years of Molecular Psychiatry may bring, we will take this opportunity to celebrate and to express our deepest gratitude to the authors, reviewers, editorial board members, and publishing staff without whom we would not exist.

\section{CONFLICT OF INTEREST}

The authors declare no conflict of interest.

$\mathrm{J}$ Licinio and M-L Wong

South Australian Health and Medical Research Institute and Department of Psychiatry, School of Medicine, Flinders University, Adelaide, Australia

E-mail: julio.licinio@sahmri.com 


\section{REFERENCES}

1 Licinio J. Molecular Psychiatry: the integration of molecular medicine and clinical psychiatry. Mol Psychiatry 1996; 1: 1-3.

2 Licinio J. Premier je suis, Second je fus, Molecular Psychiatry ne change. Mol Psychiatry 2010; 15: 777.

3 Licinio J, Wong M-L. Pharmacogenomics: The Search for Individualized Therapies. John Wiley \& Sons, 2009.

4 The White House Office of the Press Secretary. Fact Sheet: President Obama's Precision Medicine Initiative, 30 January 2015 http://www.whitehouse.gov/thepress-office/2015/01/30/fact-sheet-president-obama-s-precision-medicine-initiative.

5 Wong ML, Dong C, Flores DL, Ehrhart-Bornstein M, Bornstein S, Arcos-Burgos M et al. Clinical outcomes and genome-wide association for a brain methylation site in an antidepressant pharmacogenetics study in Mexican Americans. Am J Psychiatry. 2014; 171: 1297-1309.

6 Wong ML, Dong C, Andreev V, Arcos-Burgos M, Licinio J. Prediction of susceptibility to major depression by a model of interactions of multiple functional genetic variants and environmental factors. Mol Psychiatry 2012; 17: 624-633.

7 Baldessarini RJ, Tondo L, Strombom IM, Dominguez S, Fawcett J, Licinio J et al. Ecological studies of antidepressant treatment and suicidal risks. Harv Rev Psychiatry 2007; 15: 133-145.

8 Milane MS, Suchard MA, Wong ML, Licinio J. Modeling of the temporal patterns of fluoxetine prescriptions and suicide rates in the United States. PLoS Med 2006; 3: e190.

9 Mastronardi C, Paz-Filho GJ, Valdez E, Maestre-Mesa J, Licinio J, Wong ML. Longterm body weight outcomes of antidepressant-environment interactions. Mol Psychiatry 2011; 16: 265-272.

10 Antelman SM, Levine J, Gershon S. Time-dependent sensitization: the odyssey of a scientific heresy from the laboratory to the door of the clinic. Mol Psychiatry 2000; 5: 350-356.

11 American Psychiatric Association. Diagnostic and Statistical Manual of Mental Disorders, Fifth Edition (DSM-5). American Psychiatric Publishing: Arlington, VA, 2013.

12 Fulford KW, Bortolotti L, Broome M. Taking the long view: an emerging framework for translational psychiatric science. World Psychiatry 2014; 13: 110-117.
13 Badcock JC, Hugdahl K. A synthesis of evidence on inhibitory control and auditory hallucinations based on the Research Domain Criteria (RDoC) framework. Front Hum Neurosci 2014; 8: 180.

14 Cuthbert BN. Research domain criteria: toward future psychiatric nosology. Asian J Psychiatr 2014; 7: 4-5.

15 Cuthbert BN, Insel TR. Toward new approaches to psychotic disorders: the NIMH Research Domain Criteria project. Schizophr Bull 2010; 36: 1061-1062.

16 Cuthbert BN, Kozak MJ. Constructing constructs for psychopathology: the NIMH research domain criteria. J Abnorm Psychol 2013; 122: 928-937.

17 Insel T, Cuthbert B, Garvey M, Heinssen R, Pine DS, Quinn K et al. Research domain criteria (RDoC): toward a new classification framework for research on mental disorders. Am J Psychiatry 2010; 167: 748-751.

18 Insel TR. The NIMH Research Domain Criteria (RDoC) Project: precision medicine for psychiatry. Am J Psychiatry 2014; 171: 395-397.

19 Morris SE, Cuthbert BN. Research Domain Criteria: cognitive systems, neural circuits, and dimensions of behavior. Dialogues Clin Neurosci 2012; 14: 29-37.

20 Sternberg EM, Licinio J. Overview of neuroimmune stress interactions. Implications for susceptibility to inflammatory disease. Ann NY Acad Sci 1995; 771: 364-371.

21 Paz-Filho G, Wong ML, Licinio J. Ten years of leptin replacement therapy. Obes Rev 2011; 12: e315-e323.

22 Paz-Filho G, Wong ML, Licinio J. The procognitive effects of leptin in the brain and their clinical implications. Int J Clin Pract 2010; 64: 1808-1812.

23 Licinio J, Wong ML. The role of inflammatory mediators in the biology of major depression: central nervous system cytokines modulate the biological substrate of depressive symptoms, regulate stress-responsive systems, and contribute to neurotoxicity and neuroprotection. Mol Psychiatry 1999; 4: 317-327.

24 Graessler J, Qin Y, Zhong H, Zhang J, Licinio J, Wong ML et al. Metagenomic sequencing of the human gut microbiome before and after bariatric surgery in obese patients with type 2 diabetes: correlation with inflammatory and metabolic parameters. Pharmacogenomics J 2013; 13: 514-522.

25 Cryan JF, O'Mahony SM. The microbiome-gut-brain axis: from bowel to behavior. Neurogastroenterol Motil 2011; 23: 187-192. 